\title{
Positive predictive factors in HIV-I patients treated with enfuvirtide plus an OB that include an active boosted PI. Preliminary FastFuz study results E Ribera*1, A Antela ${ }^{2}$, JA Garcia Henarejos'3, JR Arribas ${ }^{4}$, JA Oteo ${ }^{5}$, M Lopez Gomez ${ }^{6}$ and E Ferrer ${ }^{7}$
}

Address: ${ }^{1}$ Hospital Valle de Hebrón, Barcelona, Spain, ${ }^{2}$ Hospital Universitario de Santiago de Compostela, Santiago de Compostela, Spain, ${ }^{3}$ Hospital Virgen del Rosell, Murcia, Spain, ${ }^{4}$ Hospital La Paz, Madrid, Spain, ${ }^{5}$ Hospital San Pedro, La RIoja, Spain, ${ }^{6}$ Hospital Virgen de las Nieves, Granada, Spain and ${ }^{7}$ Hospital de Bellvitge, Barcelona, Spain

* Corresponding author

from Ninth International Congress on Drug Therapy in HIV Infection

Glasgow, UK. 9-13 November 2008

Published: 10 November 2008

Journal of the International AIDS Society 2008, I I (SuppI I):P44 doi:I0.I I86/I758-2652-II-SI-P44

This abstract is available from: http://www.jiasociety.org/content/II/SI/P44

(c) 2008 Ribera et al; licensee BioMed Central Ltd.

\section{Background}

TORO studies defined four positive predictive factors (PPF) at baseline: viral load (VL) $<100,000$ copies/mL, CD4+ cell count $>100$ cells $/ \mathrm{mL}$, previous experience to $<10 \mathrm{ARVs}$, and $>2$ active drugs in the ARV regimen. The most important of these factors ( $>2$ active drugs) was an inclusion criteria in this study.

\section{Methods}

Cross-sectional, multicentre study to redefine the PPF of response in clinical practice after 12 weeks of ARV therapy, with ENF and at least one active boosted IP.

\section{Summary of results}

A total of 146 HIV-1-infected patients with viral failure were evaluated. Patients were mainly male $(75.3 \%)$, median age 42 years. Median time of ENF treatment was 13 weeks. Median baseline characteristics: VL $4.28 \log 10$ cop/mL, CD4 cell count 191 cells/mL, four previous IPs and four previous RTIs. TPV/r and DRV/r was used by $40.4 \%$ and $35.6 \%$ of patients, respectively. Patients with CD 4 count $>100$ cells $/ \mathrm{mL}$ were $74 \%$ and patients viral load (VL) $<100,000$ copies $/ \mathrm{mL} 83.6 \%$. Patients with two or more active drugs in OBT were $63.2 \%$ and with previous experience to $<10 \mathrm{ARVs}$ were $67.6 \%$. Overall response defined by protocol (decrease 1 log at week 12 or $\mathrm{VL}<50$ $\mathrm{cop} / \mathrm{ml}$ ) was $88.7 \%$. Median increase in CD4 cells count was 67 cells/mL. PPF related to $\mathrm{VL}<50$ copies $/ \mathrm{mL}$ were baseline $\mathrm{VL}<100,000$ copies/mL $(\mathrm{p}<0.005)$ and baseline CD4>100 cells/mL $(\mathrm{p}<0.05)$.

\section{Conclusion}

Enfuvirtide plus one active boosted PI led to a high percentage of response $(88.8 \%)$ with $57.5 \%$ of patients reaching virological suppression $<50$ copies/mL after 12 weeks of treatment. PPF related to response were $\mathrm{VL}<100,000$ copies $/ \mathrm{mL}$ and $\mathrm{CD} 4>100$ cells/mL. These results reinforce the idea of rescuing failing patients early (with high CD4 cells and low VL) and with at least two active drugs. 